\title{
HENSELIAN RINGS AND WEIERSTRASS POLYNOMIALS
}

\author{
BUDH NASHIER \\ (Communicated by Louis J. Ratliff, Jr.) \\ Dedicated to Professor Shreeram S. Abhyankar on his sixtieth birthday
}

\begin{abstract}
We give two characterizations of a one-dimensional Henselian domain. If $(A, \mathscr{K})$ is a local domain of Krull dimension at least two, or if $(A, \mathscr{M})$ is a one-dimensional Henselian local domain, then a polynomial $f$ in $A[T]$ is Weierstrass if and only if $(\mathscr{K}, T)$ is the only maximal ideal of $A[T]$ that contains $f$.
\end{abstract}

\section{INTRODUCTION}

Let $(A, \mathscr{M})$ be a local ring. A polynomial $f$ in the polynomial ring $A[T]$ is called a Weierstrass polynomial if $f$ is monic and all the other coefficients of $f$ are in $\mathscr{M}$. A Weierstrass polynomial in $A[T]$ is contained in only one maximal ideal of $A[T]$; namely, $(\mathscr{M}, T)$. Does this property characterize Weierstrass polynomials? The answer to this question is affirmative in the case when $A$ is a domain of Krull dimension at least two. In the one-dimensional case, we find a connection between Weierstrass polynomials and Henselian rings. As an interesting application of this connection, we prove that if $A$ is a Henselian ring and if $M$ is a maximal ideal in the Laurent polynomial ring $A\left[T, T^{-1}\right]$, then either $M \cap A[T]$ or $M \cap A\left[T^{-1}\right]$ is a maximal ideal.

In the following section, we prove two theorems giving characterizations for one-dimensional Henselian domains. In $\S 3$, we prove results about Weierstrass polynomials and establish a connection between them and Henselian domains.

For terminology, my standard source is Nagata [2]. All rings are assumed to be commutative and Noetherian (unless specified otherwise) with identity. By the dimension of a ring we mean the Krull dimension.

\section{One-Dimensional Henselian domains}

In this section, we give two characterizations of a one-dimensional Henselian domain.

Received by the editors April 23, 1990 and, in revised form, May 10, 1990; the contents of this paper were presented to the Third Mid-Atlantic Algebra Conference, held at Virginia Polytechnic Institute and State University in Blacksburg, Virginia, Saturday, April 28, 1990.

1980 Mathematics Subject Classification (1985 Revision). Primary 13B25, 13F20, 13 J15. 
Theorem 2.1. Let $A$ be a Noetherian domain. Then the following are equivalent.

(1) $A$ is a one-dimensional Henselian ring.

(2) If $B$ is a domain ( $\neq$ field) such that $B$ contains $A$, and that the quotient field of $B$ is a finite extension of the quotient field of $A$, then $B$ is integral over $A$.

Proof. (1) $\Rightarrow(2)$. Let $K$ denote the quotient field of $A$ and let $L$ denote that of $B$. Let $A^{\prime}$ and $B^{\prime}$ denote the integral closures of $A$ and $B$, respectively, in $L$. Since $A \subseteq B$, we have $A^{\prime} \subseteq B^{\prime}$. Since $L$ is a finite extension of $K$ and $A$ is a one-dimensional Noetherian domain, we have that $A^{\prime}$ is one-dimensional Noetherian by the Krull-Akizuki Theorem [2, 33.2]. We note the quotient field of $A^{\prime}$ is $L$ and that $A^{\prime}$ is a normal domain. Since $A$ is a Henselian ring, $A^{\prime}$ is local $[2,30.5]$. Thus, $A^{\prime}$ is a discrete valuation ring [3, Proposition 3, p. 7]. As $B$ is not a field, $B^{\prime} \neq L$. Thus we have $A^{\prime}=B^{\prime}$. Hence $B$ is integral over $A$, as desired.

(2) $\Rightarrow(1)$. First let us observe that $A$ is one-dimensional local. Let $P$ be a prime ideal of $A$ of height 1 . The given hypothesis yields that $A \subseteq A_{P}$ is an integral extension. Hence it follows that the dimension of $A$ is one, and that the elements of $A$ outside $P$ are units in $A$. Thus, $A$ is a local ring with $P$ as its maximal ideal. Suppose that $A$ is not Henselian. Then there exists a domain $R$ such that $A \subseteq R$ is an integral extension and that $R$ is not quasilocal $[2,43.12]$. Replacing $R$ by its derived normal ring, we may assume that $R$ is normal. Since $R$ is not a valuation ring, we can pick an element $a$ in the quotient field of $R$ such that neither $a$ nor $1 / a$ is in $R$. Set $L=K[a]$, where $K$ is the quotient field of $A$. Then, since $A[a]$ is not integral over $A$, we have $A[a]=L$. Hence it follows that $1 / a$ is integral over $A$. Thus, $1 / a \in R$, a contradiction. Therefore, $A$ is Henselian.

Theorem 2.2. Let $A$ be a one-dimensional local domain. Then $A$ is Henselian if and only if $A^{\prime}$ (the derived normal ring of $A$ ) is a discrete valuation ring such that if $f \in A^{\prime}[T]$ is an irreducible polynomial of degree $\geq 1$, then either $f$ is monic or $f(0)$ is a unit in $A^{\prime}$.

Proof. Suppose that $A$ is Henselian. Then $A^{\prime}$ is a normal local domain of dimension 1. Thus, $A^{\prime}$ is a discrete valuation ring [3, Proposition 3, p. 7]. Let $f \in A^{\prime}[T]$ be an irreducible polynomial of degree $\geq 1$. If $(f)$ is a maximal ideal in $A^{\prime}[T]$, then $(f, \pi)=A^{\prime}[T]$ where $\pi$ is a uniformizing parameter of $A^{\prime}$. Thus, $(f)$ contains an element $g \in A^{\prime}[T]$ such that $g(0)$ is a unit in $A^{\prime}$. Hence it follows that $f(0)$ is a unit in $A^{\prime}$. Suppose that $(f)$ is not a maximal ideal in $A^{\prime}[T]$. Then $A^{\prime}[T] /(f)$ is an integral extension of $A$ by Theorem 1.2, hence that of $A^{\prime}$. Therefore, $f$ must be a monic polynomial.

Conversely, suppose that $A^{\prime}$ is a discrete valuation ring with the given property for irreducible polynomials in $A^{\prime}[T]$. In order to prove that $A$ is Henselian, we show that if $R$ is a domain such that $A \subseteq R$ is an integral extension, then $R$ is quasilocal. So, let $A \subseteq R$ be an integral extension. We show that $R^{\prime}$, 
the derived normal ring of $R$, is a valuation domain. Then we would have that $R$ is quasilocal. Let $a \neq 0 \in q(R)$, the quotient field of $R$. Then $a$ is algebraic over $A$, a fortiori, over $A^{\prime}$. Let $f$ be an irreducible polynomial in $A^{\prime}[T]$ satisfied by $a$. If $f$ is monic, then $a$ is integral over $A^{\prime}$, and hence over $A$. Thus $a \in R^{\prime}$. If $f(0)$ is a unit, then $1 / a$ is integral over $A^{\prime}$ and we conclude that $1 / a \in R^{\prime}$. Thus, $R^{\prime}$ is a valuation domain, and the proof is complete.

\section{WeIERSTRASS POLYNOMIALS}

Let $(A, \mathscr{M})$ be a quasilocal ring. A monic polynomial $f \in A[T]$ is called a Weierstrass polynomial if $f=T^{n}+a_{1} T^{n-1}+\cdots+a_{n}$, where each $a_{i} \in \mathscr{M}$.

If $f$ is a Weierstrass polynomial then $(\mathscr{M}, T)$ is the only maximal ideal of $A[T]$ that contains $f$. It is easy to verify that if $(\mathscr{M}, T)$ is the only maximal ideal of $A[T]$ that contains the monic polynomial $f$, then $f$ must be a Weierstrass polynomial. However, there may be nonmonics $f \in A[T]$ such that $(\mathscr{M}, T)$ is the only maximal ideal of $A[T]$ that contains $f$. For instance, let $(A, \mathscr{M})$ denote the local ring $K[X]_{(X)}$, where $X$ is an indeterminate over the field $K$. Then the maximal ideal $(\mathscr{M}, T)$ is the only maximal ideal of $A[T]$ that contains the irreducible element $f=T^{2} X+T+X$ in $A[T]$. This observation leads us to the following definition.

Definition. Let $(A, \mathscr{M})$ be a quasilocal ring. Let us call a polynomial $f$ in $A[T]$ pseudo-Weierstrass if $(\mathscr{M}, T)$ is the only maximal ideal of $A[T]$ that contains $f$.

Clearly a Weierstrass polynomial is pseudo-Weierstrass. We have observed that a monic pseudo-Weierstrass polynomial is Weierstrass.

Let $(A, \mathscr{M})$ be a local domain and let $A^{\prime}$ denote its derived normal ring. The Mori-Nagata Theorem [2,33.10] states that $A^{\prime}$ is a quasi-semi-local Krull domain and all the maximal ideals in $A^{\prime}$ lie over $\mathscr{M}$. Let us denote the maximal ideals in $A^{\prime}$ by $\mathscr{M}_{1}, \ldots, \mathscr{M}_{r}$. Then $A^{\prime}[T]$ is an integral extension of $A[T]$ and $\left(\mathscr{M}_{1}, T\right), \ldots,\left(\mathscr{M}_{r}, T\right)$ are the only maximal ideals of $A^{\prime}[T]$ that lie over $(\mathscr{M}, T)$. It is easy to check that $f \in A[T]$ is a pseudo-Weierstrass polynomial if and only if $\left(\mathscr{M}_{1}, T\right), \ldots,\left(\mathscr{M}_{r}, T\right)$ are the only maximal ideals of $A^{\prime}[T]$ that contain $f$. Let us specialize this situation to the case when $A$ is a onedimensional local domain. Then $A^{\prime}$ is a semilocal Dedekind domain. Thus, $A^{\prime}$ is a principal ideal domain [3, Corollary, p. 12] with nonzero prime ideals $\mathscr{M}_{1}, \ldots, \mathscr{M}_{r}$. There are two types of maximal ideals in $A^{\prime}[T]$; the ones of height 2 contain some $\mathscr{M}_{i}$, and the others are principal. Note that if $f \in A^{\prime}[T]$ is a generator of a height one maximal ideal in $A^{\prime}[T]$, then $f(0)$ is a unit in $A^{\prime}$. Using this notation, in the next couple of propositions we give a complete description of pseudo-Weierstrass polynomials over Noetherian domains.

Proposition 3.1. Let $(A, \mathscr{M})$ be a local domain of dimension $>1$. Then pseudoWeierstrass polynomials in $A[T]$ are precisely the Weierstrass ones. 
Proof. Let $f=a_{0} T^{n}+a_{1} T^{n-1}+\cdots+a_{n} \in A[T]$ be a pseudo-Weierstrass polynomial. We shall show that $a_{0} \notin \mathscr{M}$. Let $\mathscr{M}_{1}$ be a maximal ideal of $A^{\prime}$ that lies over $\mathscr{M}$. Set $R=A_{\mathscr{M}_{1}}^{\prime}$. Then we have that $f$ is a pseudo-Weierstrass polynomial in $R[T]$. Note that $R$ is a Krull ring. If we show that $a_{0} \notin \mathscr{M}_{1}$, then we will have that $a_{0} \notin \mathscr{M}$. Thus, for our proof, we may as well assume that $(A, \mathscr{M})$ is a quasilocal Krull domain. Let us suppose that $a_{0} \in \mathscr{M}$. Since $(A, \mathscr{M})$ is a quasilocal Krull domain of dimension $>1$, we can find an element $p \in \mathscr{M}$ such that $a_{0}$ is not a zero-divisor modulo $p A$. Then no element of the set $a_{0}+p A$ is a unit in the ring $A[1 / p]$. Now it is easy to check that the ideal $I=(f, 1-p T)$ is a proper ideal of $A[T]$. Thus $I$, whence $f$, is contained in a maximal ideal, necessarily distinct from $(\mathscr{M}, T)$, of $A[T]$, a contradiction. Therefore $a_{0} \notin \mathscr{M}$. Thus $f$ is monic, and hence Weierstrass. Since a Weierstrass polynomial is pseudo-Weierstrass, the proof is complete.

Proposition 3.2. Let $(A, \mathscr{M})$ be a local domain of dimension one. Then a nonconstant polynomial $f \in A[T]$ is pseudo-Weierstrass if and only if all but one of the coefficients of $f$ are in $\mathscr{M}$ and if $g$ is an irreducible factor of $f$ in $A^{\prime}[T]$ then $g(0)$ is not a unit in $A^{\prime}$.

Proof. Suppose that $f \in A[T]$ is a pseudo-Weierstrass polynomial. Then clearly $f(0) \in \mathscr{M} A$. Since $(T+\mathscr{M} A[T])$ is the only prime ideal in $[A / \mathscr{M}][T]$ that contains the element $f+\mathscr{M} A[T]$, it follows that all but one of the coefficients of $f$ are in $\mathscr{M}$. Suppose $g$ is an irreducible factor of $f$ in $A^{\prime}[T]$. If $g(0)$ is a unit in $A^{\prime}$, then either $(g)$ is a maximal ideal or is contained in the maximal ideal of $A^{\prime}[T]$ different from the ones that lie over $(\mathscr{M}, T)$. In either case, we conclude that $f$ is contained in a maximal ideal in $A[T]$ other than $(\mathscr{M}, T)$, a contradiction. Hence, $g(0)$ is not a unit.

Conversely, suppose that $f$ has the property described above. We observe that $f(0) \in \mathscr{M}$. Thus, $f \in(\mathscr{M}, T)$. Suppose that $M$ is a maximal ideal in $A[T]$ that contains $f$. If $\operatorname{ht}(M)=2$, then $M$ contains $\mathscr{M}$. As exactly one coefficient of $f$ is a unit, we have that $T \in M$, and hence $M$ is $(\mathscr{M}, T)$. Suppose that $\operatorname{ht}(M)=1$. Let $M^{\prime}$ be a maximal ideal in $A^{\prime}[T]$ that lies over $M$. Then $M^{\prime}$ has height one. If $g$ is a generator of $M^{\prime}$ then $g(0)$ is a unit in $A^{\prime}$ and $g$ is a factor of $f$, a contradiction. Thus, $(\mathscr{M}, T)$ is the only maximal ideal in $A[T]$ that contains $f$, and so $f$ is a pseudo-Weierstrass polynomial.

Corollary 3.3. Let $(A, \pi)$ be a discrete valuation ring. Then an irreducible polynomial $f \in A[T]$ is pseudo-Weierstrass if and only if $f(0) \in \pi A$, and exactly one coefficient of $f$ is a unit. A reducible polynomial $f \in A[T]$ is pseudoWeierstrass if and only if each irreducible factor of $f$ is so.

We already noticed that not every one-dimensional domain $A$ enjoys the property that every pseudo-Weierstrass polynomial in $A[T]$ is Weierstrass. Now we show that a Henselian domain enjoys this property.

Proposition 3.4. Let $A$ be a Henselian domain of dimension one. Then a pseudoWeierstrass polynomial in $A[T]$ is Weierstrass. 
Proof. Let $f$ be a pseudo-Weierstrass polynomial in $A[T]$. Let $A^{\prime}$ be the derived normal ring of $A$. Then $A^{\prime}$ is a discrete valuation ring and we note that $f$ is a pseudo-Weierstrass polynomial in $A^{\prime}[T]$. By Corollary 3.3, each irreducible factor of $f$ is pseudo-Weierstrass. Thus, each irreducible factor of $f$ is monic in $A^{\prime}[T]$ by Theorem 2.2. Hence $f$ is monic in $A^{\prime}[T]$. Since $A^{\prime}$ is integral over $A, f$ is monic in $A[T]$. Thus $f$ is Weierstrass.

Proposition 3.5. Let $A$ be a Henselian domain of dimension one. Then for any maximal ideal $M$ in $A\left[T, T^{-1}\right]$, either $M \cap A[T]$ or $M \cap A\left[T^{-1}\right]$ is a maximal ideal.

Proof. If $A^{\prime}$ is the derived normal ring of $A$, then it suffices to prove that for any maximal ideal $M$ in $A^{\prime}\left[T, T^{-1}\right]$ either $M \cap A^{\prime}[T]$ or $M \cap A^{\prime}\left[T^{-1}\right]$ is a maximal ideal. Thus, we may replace $A$ by its derived normal ring and assume that $A$ is a Henselian discrete valuation ring. If $h t(M)=2$, then both $M \cap A[T]$ and $M \cap A\left[T^{-1}\right]$ are maximal. Suppose that $\operatorname{ht}(M)=1$. Then $M \cap A[T]=(g)$ for some irreducible polynomial $g$ of degree $\geq 1$ in $A[T]$. If $(g)$ is a maximal ideal in $A[T]$ then there is nothing more to prove. Otherwise, we note that $g$ is a pseudo-Weierstrass polynomial. By Proposition 3.4, $g$ is Weierstrass. Then we observe that $M \cap A\left[T^{-1}\right]$ is a maximal ideal.

Remark. It would be a very nice theorem to prove that a one-dimensional local domain is Henselian by assuming that every maximal ideal in $A\left[T, T^{-1}\right.$ ] contracts to a maximal ideal in $A[T]$ or in $A\left[T^{-1}\right]$. It is not hard to prove that the above hypothesis implies at least that $A^{\prime}$, the derived normal ring of $A$, is local and hence a discrete valuation ring. To see this, let $p_{1}, p_{2}, \ldots, p_{r}$ be all the distinct (nonassociate) primes in $A^{\prime}$. Suppose that $r>1$. Let $a_{0}=p_{1} p_{2} \cdots p_{r}$. For each $i=1,2, \ldots, r$, let $a_{i}=p_{1} p_{2} \cdots p_{i-1} p_{i+1} \cdots p_{r}$. Let $f=a_{0}+a_{1} T+\cdots+a_{r} T^{r}$. By Eisenstein's criterion, $f$ is an irreducible polynomial in $A^{\prime}[T]$. We observe that the prime ideal $f A^{\prime}[T]$ is contained only in the maximal ideals $\left(p_{i}, T\right), i=1,2, \ldots, r$. Thus, $M=f A^{\prime}\left[T, T^{-1}\right]$ is a maximal ideal in $A^{\prime}\left[T, T^{-1}\right]$, and we have that neither $M \cap A^{\prime}[T]$ nor $M \cap A^{\prime}\left[T^{-1}\right]$ is a maximal ideal. By letting $P=M \cap A\left[T, T^{-1}\right]$, we obtain a maximal ideal in $A\left[T, T^{-1}\right]$ such that its contractions to $A[T]$ and $A\left[T^{-1}\right]$ are not maximal. Thus, $r=1$ and $A^{\prime}$ is a discrete valuation ring. In the case of a discrete valuation ring, a proof of the converse of Proposition 3.5 would yield one for the converse of Proposition 3.4, and vice versa, as is shown below.

Theorem 3.6. Let $A$ be a discrete valuation ring. Then for every maximal ideal $M$ in $A\left[T, T^{-1}\right]$, either $M \cap A[T]$ or $M \cap A\left[T^{-1}\right]$ is a maximal ideal if and only if every pseudo-Weierstrass polynomial in $A[T]$ is Weierstrass.

Proof. Suppose that every pseudo-Weierstrass polynomial in $A[T]$ is Weierstrass. Let $M$ be a maximal ideal in $A\left[T, T^{-1}\right]$. To show that either $M \cap A[T]$ or $M \cap A\left[T^{-1}\right]$ is maximal, we need to consider only the situation when $\operatorname{ht}(M)=1$. Then $M \cap A[T]=(g)$, where $g$ is an irreducible polynomial 
of degree $\geq 1$ in $A[T]$. Suppose that the prime ideal $(g)$ is not maximal in $A[T]$. Since $g A\left[T, T^{-1}\right]=M$ is maximal, we observe that $g$ is a pseudoWeierstrass polynomial. By hypothesis, $g$ is Weierstrass. Since $g$ is an irreducible Weierstrass polynomial in $A[T]$, we have that $M \cap A\left[T^{-1}\right]$ is a maximal ideal.

Conversely, suppose that $f \in A[T]$ is a pseudo-Weierstrass polynomial. We may assume that $f$ is irreducible. Then the prime ideal $f A\left[T, T^{-1}\right]$ is a maximal ideal. Since $f A[T]$ is not maximal, we have $f A\left[T, T^{-1}\right] \cap A\left[T^{-1}\right]$ is a maximal ideal of height one in $A\left[T^{-1}\right]$. As $g=T^{-\operatorname{deg}(f)} f$ is a generator of a height 1 maximal ideal in $A\left[T^{-1}\right]$, we have that $g(0)$ is a unit in $A$. Hence $f$ is monic in $A[T]$. Therefore, $f$ is a Weierstrass polynomial, as required.

Let us note that if $A$ is a Henselian local ring, then $A / I$ also is a Henselian local ring for any ideal $I$ in $A$. The final result of the paper is the following theorem.

Theorem 3.7. Let $A$ be a Henselian local ring. Then for every maximal ideal $M$ in $A\left[T, T^{-1}\right]$, either $M \cap A[T]$ or $M \cap A\left[T^{-1}\right]$ is a maximal ideal.

Proof. Let $P=M \cap A$. By a result of Artin and Tate [1], it follows that $\operatorname{dim}(A / P) \leq 1$. We go modulo $P$, and then obtain the desired result via Proposition 3.5.

Added in proof. The question posed in the remark has been answered affirmatively by Bill Heinzer, Dave Lantz, and Sylvia Wiegand and, independently, by the author.

\section{ACKNOWLEDGMENT}

I am grateful to the referee and to Professor Gerd Faltings of Princeton University for pointing out a flaw in an earlier proof of Proposition 3.1.

\section{REFERENCES}

1. E. Artin and J. Tate, A note on finite integral extensions, J. Math. Soc. Japan 3 (1951), 74-77.

2. M. Nagata, Local rings, Interscience, New York, 1962.

3. J. P. Serre, Local fields, Graduate Texts in Math., vol. 67, Springer-Verlag, New York, Heidelberg, and Berlin, 1979.

Department of Mathematics, Florida State University, Tallahassee, Florida 323063027 